\title{
Lessons learned from the multidisciplinary UK National Flap Registry initiative and plans for the future
}

\author{
Michael Ho ${ }^{1 \wedge}$, Andrew Schache ${ }^{2}$, Michael Nugent ${ }^{3}$, Anita Hazari $^{4}$ \\ ${ }^{1}$ Oral and Maxillofacial Surgery, Leeds Dental Institute, Leeds, UK; ${ }^{2}$ Liverpool Head and Neck Centre, University of Liverpool, Liverpool, UK; \\ ${ }^{3}$ Oral and Maxillofacial Surgery, Sunderland Royal Hospital, Sunderland, UK; ${ }^{4}$ Plastic and Reconstructive Surgery, Queen Victoria Hospital, East \\ Grinstead, UK \\ Contributions: (I) Conception and design: All authors; (II) Administrative support: All authors; (III) Provision of study materials or patients: All \\ authors; (IV) Collection and assembly of data: All authors; (V) Data analysis and interpretation: All authors; (VI) Manuscript writing: All authors; (VII) \\ Final approval of manuscript: All authors. \\ Correspondence to: Michael Ho. Consultant Oral and Maxillofacial Surgeon, Oral and Maxillofacial Surgery, Leeds Dental Institute, Clarendon Way, \\ LS2 9LU, Leeds, UK. Email: m.ho@leeds.ac.uk.
}

\begin{abstract}
The conception of the UK National Flap Registry (UKNFR) came from the political climate which had arisen following clinical outcome publications by the NHS Commissioning Board for surgical specialties during 2012 and 2013, in the United Kingdom. In 2013, a proposal was made to British Association of Plastic, Reconstructive and Aesthetic Surgeons (BAPRAS), with the recommendation that a web-based software platform would be developed to develop a national flap register. The emphasis was on establishing a database that could be accessed and used by any speciality performing reconstructive surgery and thereby encouraging multidisciplinary collaboration, including the British Association of Oral and Maxillofacial Surgeons (BAOMS), British Association of Head and Neck Oncologists (BAHNO), Association of Breast Surgery (ABS) and British Society for Surgery of the Hand (BSSH). UKNFR is the first national registry of this type in the world to collect data on all major pedicled and free flap operations. This review article summarises the history of the UKNFR, the main findings of its first report published in December 2019 and lessons learned along the journey of quality improvement. Suggestions for future development and opportunities for an international collaborative approach in the registration of flap reconstruction in the head and neck region are discussed.
\end{abstract}

Keywords: Quality improvement; head and neck reconstruction; flap registry

Received: 01 January 2021; Accepted: 24 December 2021; Published online: 11 January 2022.

doi: $10.21037 /$ fomm-21-2

View this article at: https://dx.doi.org/10.21037/fomm-21-2

\section{Introduction}

The concept of quality improvement which encompasses clinical audit, quality assurance and performance measurement have been increasingly accepted and incorporated into surgical practice over the last two decades. The drive for the formation of the UK National Flap Registry (UKNFR) came from the political climate surrounding clinical outcome publications by the National Health Service (NHS) Commissioning Board for surgical specialties during 2012 and 2013, in the United Kingdom. All ten specialties named by the NHS Commissioning Board had national registries supported either by public funds through the Healthcare Quality Improvement Partnership (HQIP) or specialist surgical associations. As the NHS moved towards a culture of assessment based

\footnotetext{
^ ORCID: 0000-0001-9810-3136.
} 
on outcome measures there was a need for clear metrics capable of defining performance reliably. These needed to support the targeting and monitoring of improvements whilst maintaining high quality care.

Mortality in head and neck reconstructive surgery is fortunately a relatively rare event. Therefore, there was a requirement for outcome measures in this aspect of clinical practice that are clinically relevant, quantifiable with scope for service improvement and above all, reflect the width and scope of practice.

\section{First steps in comparative flap surgery audit}

As far back as 1999, there was support for the idea that major flap surgery outcomes be utilised as a monitor and/ or indicator of success for reconstructive surgery with the endpoint being flap survival and other indicators such as reoperation rate, length of hospital stay and functional outcomes. A systematic and transparent clinical audit of outcomes is instrumental in their professional development and revalidation for most surgeons performing complex surgery, as part of a holistic quality improvement programme framework. The Clinical Effectiveness Subcommittee (CESC) which was set up by British Association of Plastic Surgeons (BAPS) in 1999 and the Clinical Effectiveness Unit (CEU) of the Royal College of Surgeons of England, identified 5 criteria for a complex surgery marker procedure, which were:

* High-volume, performed in all reconstructive surgery units;

* Representative of the range of challenges within reconstructive surgery;

* Must have frequent outcome events;

* Have widely accepted and easily measured patient risk factors;

* Have reliable and valid outcome measures.

Free and pedicled flaps were selected as the appropriate marker procedures. Although these flaps may have different anatomical donor and recipient sites, flap composition and indications, all these procedures share the same objective and desirable outcomes of high rates of flap survival, minimal unplanned rates of re-operation during the same admission for complications of the flap surgery and, achieving the optimal aesthetic and functional outcomes for which they were intended in the care of patients. Free and large pedicled flaps constitute a major proportion of the workload of all reconstructive surgery units. Risk factors were identified, based on evidence from the published literature (1) and clinical practice, that influence flap survival rates, increase complication rates and prolong hospital length of stay. These included smoking, previous radiotherapy, body mass index and ASA grade. A registry was designed which included these risk factors and also the flap type, composition, operation time, and operator grade.

A 3-month pilot study involving 5 units was published in JPRAS 2006 (2) reporting a case-mix reflecting the unit workload of $41 \%$ flaps to the trunk including breast reconstruction, $25 \%$ to the head and neck, $25 \%$ to lower limb and $8 \%$ to the upper limb. The pilot study was presented to BAPRAS and the European Society of Plastic, Reconstructive and Aesthetic Surgeons (ESPRAS) demonstrating the acceptability to surgeons of the format with good overall outcomes for flap survival across all units participating in the study.

\section{Proposal for a national flap registry}

In 2013, a formal proposal was made to BAPRAS following the same principles as the CESC/CEU design brief, with the recommendation that a web-based software platform would be developed. The emphasis was on establishing a database that could be used by any speciality performing reconstructive surgery and thereby encouraging collaboration, including the British Association of Oral and Maxillofacial Surgeons (BAOMS), British Association of Head and Neck Oncologists (BAHNO), Association of Breast Surgery (ABS) and British Society for Surgery of the Hand (BSSH). In 2014 to 2015, the political climate was further concentrated on Consultant Outcomes Publications, driven by NHS England and Healthcare Quality Improvement Partnership (HQIP). Thirteen surgical specialties published their outcome data as the flap registry was being developed.

\section{Web-based software}

In a fast evolving and ever-changing digital world, it was suggested that the approach to clinical data collection utilising of web-based tools, as opposed to a traditional software route, would ensure wider uptake amongst surgeons and optimally locate the interface for future refinements. Several factors relevant to this project influenced this recommendation:

* Speed of iteration: web-based software exists in a single remote location, allow updates to 
be implemented out with immediate effect, eliminating the need to distribute software updates on devices or downloads.

* Monitoring: allows collection and collation of usage information from all usage of a system, allowing for further development decisions to be made in the light of real usage patterns.

* Device compatibility: software delivered through a web browser is compatible with any device that runs a web browser, from a mobile tablet to a PC.

* Mobile optimized: web-based software can adapt its layout and interface to suit different screen sizes and orientations, creating an optimal experience whichever device the tool is being used.

* Best practice reporting: depending on the need, several methods for reporting and visualising data can be incorporated into web-based tools.

* Ease of distribution: surgeons in different hospitals can start to use the new tools straight away without the need for explicit distribution, which keeps costs down but also allows for groups to trial the tools without the need for central organization.

* Simplified maintenance: centralised software keeps maintenance costs down due to only having one system to maintain. Patient data security was the most challenging task, since the web-based data would need to be stored offsite.

* BAPRAS had three important requirements for the registry:

- It needed to be built for a mobile platform, so that data could be entered through a smart device or tablet, in addition to conventional personal computers.

- It had to be based on a secure platform hosting confidential patient data compliant with the stipulations of the Data Protection Act 1998 and subsequently General Data Protection Regulation (GDPR) 2018.

- The data had to be stored safely within the United Kingdom, with enough primary and secondary backup server facilities.

\section{Structure of the registry}

Because the UKNFR dataset needed to include all anatomical areas, the registry design was complex, in effect several registries built within one larger one. After a rigorous procurement process, Dendrite Clinical Systems, a specialist company with a track record in this field was chosen to develop the required platform. Following agreement by BAPRAS Council, the UKNFR database was designed by clinicians based on the online CEU proforma derived database together with elements from other similar systems from units including Oxford, Chelmsford, East Grinstead and Liverpool.

The registry was designed with the support of Dendrite Clinical Systems, alongside an Editorial published in 2015 in the Fournal of Plastic Reconstructive and Aesthetic Surgery ( $P$ PRAS) outlining the principles of the Registry (3). UKNFR went live on 1st August 2015 when the first NHS patient flap reconstruction case was entered into the registry.

\section{Surgeon dashboard}

One of the big successes of the registry has been the surgeon dashboard: the registry displays the surgeon's individual data on a dashboard which allows easy visualisation in real time. This includes the number of procedures performed, case-mix, flap survival, unplanned return to theatre and length of hospital stay. The dashboard has critical importance and the output will be analysed during the UK NHS consultant appraisal and revalidation process, as it produces evidence of the surgeon's performance in the format of a real time audit. Prior to producing a copy of the database output, all consultants were informed that they should ensure that all of their consecutive patient records should be uploaded, retrospectively if applicable, and be accurate. This would help to reduce issues of sample bias and case ascertainment. It has been recognised in the preparation of the 2019 UKNFR report that future additional work will be required to ensure that data collection from each unit has been complete and is therefore truly representative.

UKNFR is the first national registry of this type in the world to collect data on all major pedicled and free flap operations. In the first report from UKNFR, a census of the database was taken on 8th August 2019. The patient records comprising the data for this first report included those treated from 1st January 2016 to 30th June 2019. The published report is accessible to all surgeons and patients internationally and we would highly suggest the readership access this document for the complete report and more detailed information (4). 


\section{Head and neck reconstruction in the first UKNFR report [summarised excerpts for the First UKNFR report 2019 (4)]}

\section{Indication for reconstruction}

The majority of head and neck reconstructive surgery using flaps was for cancer patients (70\%). Although some were delayed secondary procedures after tumour ablation, most were performed primarily (immediately). The most common diagnosis was squamous cell carcinoma (SCC).

\section{Demographics}

The proportion of patients over the age of 64 years (the average age for patients having a head \& neck procedure) varied slightly between hospitals. There were a relatively greater number of men in this group of patients, but the measures of average age seem to be largely independent of gender. A separate analysis showed that men were overrepresented in the $<40$-year-old age group, and there was a greater proportion of women over the age of 79 .

\section{Co-existing conditions and disease profile}

Risk factors including smoking/tobacco use and alcohol consumption were included with pulmonary disease, diabetes, ischaemic heart disease, peripheral vascular disease and hypertension as the main data fields in relation to coexisting conditions and disease profile.

\section{Type and number of flaps}

Almost all of the operations involved just one flap being transferred to one recipient defect. Common flaps used for head and neck reconstruction were the radial forearm flap (RFF) and the anterolateral thigh (ALT) flap. The most frequently used flap for mandibular reconstruction was the free fibula (often with a fasciocutaneous component).

\section{Recipient sites}

The recipient sites for head \& neck reconstruction were divided into internal and external sites in the database. These sites are further subdivided according to anatomical subunits. The majority of reconstructions were for internal sites such as the oral cavity or mandible. The most common primary tumour sites were the tongue and the mandible, followed by floor of mouth.
The commonest external sub-units for flap reconstruction were middle third of face excluding maxilla and the neck. For internal sub-units, tongue, mandible and floor of mouth were the commonest.

\section{Microvascular anastomosis}

Venous couplers were used in $58 \%$ of end-to-end vein anastomoses with reported patency rates of $98.8 \%$. Most microvascular anastomoses have conventionally been handsewn, however in the last decade, there has been an increased uptake of the venous coupler by microsurgeons due to its ease of use, reduced operative time and reliability (5).

\section{Flap survival}

There was uniformly high flap survival (94\% overall survival, complete and/or partial flap failures are included as one category in this version of the UKNFR) except where free and pedicled flaps were combined, but these numbers in this sub-group were too small to provide a conclusive result. Those cases where combinations of flaps were used usually represent larger or more complex defects. Flap survival rates were high regardless of the presence of specific risk factors or co-existing conditions. Some patients who experienced a partial flap failure may not have required a further reconstructive procedure. However, if partial flap loss was clinically significant then further analysis of any re-operations to the recipient site, such as whole of flap removed or part of flap removed, would provide more detail.

Similarly, a second flap to the same recipient site might have been recorded. If no re-operation was required, then that partial failure can be taken to have been of less clinical significance. This means that rather than recording an estimated percentage of tissue loss for an individual flap, an evaluation of the real clinical impact can be used to analyse the outcome. In subsequent iterations of UKNFR from January 2020 and for all future reports, a classification of flap reconstructive outcomes has been adopted that distinguishes between the different levels of flap survival and the need for a second flap or prosthesis, all of which could impact on patient recovery (6). With the onset of the SARS-CoV-2 pandemic, data points relating to COVID-19 testing pre-operatively, at discharge and onset of symptoms within 2 weeks of discharge have also been included.

Variation in the flap survival rates between contributing hospitals were apparent, however most of these differences were not statistically significant. There is only one hospital that reports a significant deviation from the database average: the surgeons at this hospital reported a significantly 
higher flap survival rate than average. In future reports, data reported from statistical outliers can be analysed through a more detailed examination of case-mix, comorbidities and risk factors. Total flap loss occurred in less than $1 \%$ of reconstructions involving the tongue, floor of mouth and mandible. However, partial flap loss was significantly greater than $1 \%$ at these sites. It is not known how many of these then necessitated a second flap reconstruction.

\section{Re-operation and length of hospital stay}

Operations requiring a combination of free and pedicled flaps together had a higher re-operation rate $(64 \% v s$. free flap only $12 \%$ vs. pedicled flap only $9 \%$ ).

The following were associated with low rates of postoperative stay in excess of 13 days:

* No history of smoking;

* ASA grade 1-2;

* Delayed reconstruction;

* External defect reconstructions.

\section{Discussion-lessons learned from the First UKNFR report 2019 (Head and Neck) and suggestions for the future}

The publication of the First UKNFR report is indeed an important milestone from the perspective of reporting clinical outcomes in reconstructive surgery. It has further reinforced the principle that multi-specialty collaborations should be considered essential for projects such as this undertaken at a national or multi-national scale.

The report forms the baseline for future findings in reconstructive surgical trends in the UK and it is hoped that these patient outcome data could be utilised as part of a quality improvement initiative. One of the challenges faced during the interpretation of data collected during the period covered by the report was risk stratification of patients based on their lifestyle risk factors, demographics, co-existing medical conditions and disease (e.g., cancer) stage. Due to some issues around data completeness and the lack of a validated model to ascertain complexity of casemix, it was not possible to compare and contrast the surgical outcomes of different centres in depth. In the next iteration of the UKNFR, it is crucial that data fields are linked to a validated risk-stratified model capable of allowing accurate assignment of patient case-mix complexity.

Several hospitals reported fewer than 10 patient cases during the study period which represented low-volume activity levels. It would be interesting to know whether these data truly reflect the totality of head and neck flap surgery at each of these hospitals. Parallel to the production of the UKNFR report which includes voluntary data entry from all of the UK and the Republic of Ireland, the BAOMS Quality Outcomes in Maxillofacial Surgery (QOMS) team engaged with the National Consultant Improvement Project (NCIP) team to formulate a surgeon's dashboard for the reporting of outcomes in head and neck cancer surgery, and reconstruction, in England. Access was granted to the Hospital Episode Statistics (HES) Database which coded and recorded each episode of surgical procedure performed through local coders in each NHS organisation. Furthermore, the BAOMS QOMS team had sought OMFS surgical units in the UK to voluntarily report their locally recorded data of surgical activity in their local registries/ database. This information has all been summarised in Table 1. Overall, 36 (52\%) of head and neck/OMFS units in England contributed information to UKNFR during the period of the first report, and from these units, $27 \%$ $(1,604)$ of the 5,871 registered cases of head and neck flap procedures registered on the HES database was captured by the UKNFR. These levels represent both a success with cross specialty engagement but also a clarion call to the majority to harness this opportunity to benchmark their clinical practice with a view towards keeping high quality patient outcomes centremost. Perhaps this is an issue that can be investigated in future reports as this goes to the heart of questions around the completeness and representativity of the data in the UKNFR. Further engagement initiatives with the head and neck surgical community or perhaps offer of support for data entry could improve the take up of this national registry. The BAHNO Standards 2020 document (in press), has recommended that the minimum indicative number of free flap cases undertaken within an individual MDT structure should exceed 25 per annum. Free flap success for all units must exceed $90 \%$, and ideally be above $95 \%$. All surgeons providing Head and Neck reconstructive services should maintain documented evidence of the number of flaps undertaken annually with associated success rates, so as to facilitate comparison within their unit/MDT structure.

All units should audit free flap outcomes annually, including:

* Number of free flap procedures undertaken;

* Returns to theatre;

* Salvage rates;

* Overall success (partial or otherwise);

* Patient length of stay. 
Table 1 Number of flap reconstruction patient cases reported through HES, UKNFR and voluntarily by OMFS units in England from 1 August 2015 to 9 August 2019

\begin{tabular}{|c|c|c|c|}
\hline \multirow{2}{*}{ Provider } & \multicolumn{3}{|c|}{ Volume } \\
\hline & HES & UKNFR & Self-reported \\
\hline 1 & 10 & & \\
\hline 2 & 10 & & \\
\hline 3 & 10 & & \\
\hline 4 & 20 & 102 & \\
\hline 5 & 35 & 2 & \\
\hline 6 & 45 & & \\
\hline 7 & 55 & 1 & \\
\hline 8 & 60 & 25 & \\
\hline 9 & 70 & & \\
\hline 10 & 75 & & \\
\hline 11 & 90 & & \\
\hline 12 & 90 & & \\
\hline 13 & 95 & 113 & \\
\hline 14 & 95 & & 82 \\
\hline 15 & 100 & 116 & 128 \\
\hline 16 & 100 & & \\
\hline 17 & 105 & 39 & \\
\hline 18 & 105 & & \\
\hline 19 & 105 & 3 & \\
\hline 20 & 110 & 14 & \\
\hline 21 & 115 & & \\
\hline 22 & 115 & 4 & \\
\hline 23 & 115 & 55 & \\
\hline 24 & 115 & 41 & \\
\hline 25 & 120 & & \\
\hline 26 & 125 & 1 & 130 \\
\hline 27 & 130 & & 144 \\
\hline 28 & 130 & & 113 \\
\hline 29 & 145 & 50 & \\
\hline 30 & 150 & & \\
\hline 31 & 150 & 8 & 191 \\
\hline 32 & 155 & & \\
\hline 33 & 160 & & \\
\hline 34 & 160 & 36 & \\
\hline 35 & 180 & 1 & \\
\hline 36 & 185 & 11 & \\
\hline 37 & 185 & 93 & \\
\hline 38 & 195 & & \\
\hline
\end{tabular}

Table 1 (continued)
Table 1 (continued)

\begin{tabular}{|c|c|c|c|}
\hline \multirow{2}{*}{ Provider } & \multicolumn{3}{|c|}{ Volume } \\
\hline & HES & UKNFR & Self-reported \\
\hline 39 & 210 & & \\
\hline 40 & 235 & & \\
\hline 41 & 235 & & \\
\hline 42 & 240 & 8 & \\
\hline 43 & 245 & & 303 \\
\hline 44 & 250 & 128 & \\
\hline 45 & 270 & 44 & \\
\hline 46 & 290 & & 184 \\
\hline 47 & 295 & & \\
\hline 48 & 310 & 4 & \\
\hline 49 & 330 & & \\
\hline 50 & 400 & 179 & \\
\hline 51 & $<8$ & 1 & \\
\hline 52 & $<8$ & 153 & \\
\hline 53 & $<8$ & & \\
\hline 54 & $<8$ & 4 & \\
\hline 55 & $<8$ & & \\
\hline 56 & $<8$ & & \\
\hline 57 & $<8$ & & \\
\hline 58 & $<8$ & & \\
\hline 59 & $<8$ & & \\
\hline 60 & $<8$ & 161 & \\
\hline 61 & $<8$ & 162 & \\
\hline 62 & $<8$ & & 0 \\
\hline 63 & $<8$ & 2 & \\
\hline 64 & $<8$ & & \\
\hline 65 & $<8$ & 1 & \\
\hline 66 & $<8$ & & \\
\hline 67 & $<8$ & 8 & \\
\hline 68 & $<8$ & 34 & \\
\hline 69 & $<8$ & & \\
\hline Total & 5,871 & 1,604 & 1,275 \\
\hline Number of units with data & 69 & 36 & 9 \\
\hline $\begin{array}{l}\text { Percentage of total number of flap } \\
\text { procedures }(\%)\end{array}$ & 100 & 27 & 22 \\
\hline $\begin{array}{l}\text { Percentage of total number of } \\
\text { institutions providing information (\%) }\end{array}$ & 100 & 52 & 13 \\
\hline
\end{tabular}

NCIP flap volumes (01/08/15 to 09/08/19)-all numbers have been rounded to the nearest 5 and all small numbers [1-7] have been replaced with " $<8$ ". HES, Hospital Episode Statistics; UKNFR, UK National Flap Registry; NCIP, National Consultant Improvement Project. 
All units should be prepared to present their outcomes following free flap reconstruction for external peer review.

Another issue which has been alluded to above is the issue of partial flap success/failure. As reported, the 6\% flap failure rate could either be an overestimate of flap failure if surgeons included partial failures which did not have any significant impact on patients in terms of care or need for further reconstruction, or misrepresentation if partial flap failures which did not require any additional intervention were included into the count of flap successes. Whilst anastomotic failures are binary events in microvascular free tissue transfer, partial flap losses can be more heterogenous in presentation and outcomes given that they impact different on patients, depending on the recipient site defect and flap design (e.g., simple vs. chimeric).

As initiatives for quality improvement gather pace with the NCIP surgeon's dashboard, UKNFR, Head and Neck Cancer Audit (HANA) and BAOMS QOMS the needs will arise for data sharing across multiple registries. This will reduce the burden of data entry to the surgical teams. It is therefore essential that the projects collaborate in ensuring that data sharing agreements are in place with the appropriate data fields to be collected in order to allow single registry data entry which would populate multiple connected and relevant registries to produce the relevant reports related to head and neck reconstruction especially in patients who have this treatment as part of their cancer care.

As the UKNFR develops and matures, it aims ultimately to collaborate at the international stage with reconstructive colleagues across the globe. An international collaboration of national flap registries would be a significant and progressive next step to improve exchange of knowledge and best practice attributes within the international community of head and neck reconstructive surgeons. The principles which underly this ambition is consistent with previous developments in orthopaedic joint registries and multinational bariatric global registry (https://www. e-dendrite.com/database-registries).

In summary, the UKNFR has formed the basis for future quality improvement initiatives in UK head and neck reconstructive surgery and will in time to come be viewed as one of several key milestones in this subspecialty area of practice.

\section{Acknowledgments}

Funding: None.

\section{Footnote}

Provenance and Peer Review: This article was commissioned by the editorial office, Frontiers of Oral and Maxillofacial Medicine, for the series "Head and Neck Reconstruction". The article has undergone external peer review.

Conflicts of Interest: All authors have completed the ICMJE uniform disclosure form (available at https://fomm. amegroups.com/article/view/10.21037/fomm-21-2/ coif). The series "Head and Neck Reconstruction" was commissioned by the editorial office without any funding or sponsorship. $\mathrm{MH}$ served as the unpaid Guest Editor of the series, and serves as an unpaid editorial board member of Frontiers of Oral and Maxillofacial Medicine from October 2019 to September 2023. The authors have no other conflicts of interest to declare.

Ethical Statement: The authors are accountable for all aspects of the work in ensuring that questions related to the accuracy or integrity of any part of the work are appropriately investigated and resolved.

Open Access Statement: This is an Open Access article distributed in accordance with the Creative Commons Attribution-NonCommercial-NoDerivs 4.0 International License (CC BY-NC-ND 4.0), which permits the noncommercial replication and distribution of the article with the strict proviso that no changes or edits are made and the original work is properly cited (including links to both the formal publication through the relevant DOI and the license). See: https://creativecommons.org/licenses/by-nc-nd/4.0/.

\section{References}

1. Ho MW, Brown JS, Magennis P, et al. Salvage outcomes of free tissue transfer in Liverpool: trends over 18 years (1992-2009). Br J Oral Maxillofac Surg 2012;50:13-8.

2. Cole RP, Browne JP. Towards performance measurement in reconstructive surgery: a multicentre pilot study of free and pedicled flap procedures. J Plast Reconstr Aesthet Surg 2006;59:257-62.

3. Hazari A, Walton P. The UK National Flap Registry (UKNFR): A National Database for all pedicled and free flaps in the UK. J Plast Reconstr Aesthet Surg 2015;68:1633-6.

4. Hazari A, Cole R, Fowler C, et al. First UK National Flap Registry Report 2019. Available online: http://www.bapras. 
org.uk/docs/default-source/default-document-library/ uknfr-first-report-4-dec-2019.pdf? sfvrsn=2http://www. bapras.org.uk/docs/default-source/default-documentlibrary/uknfr-first-report-4-dec-2019.pdf?sfvrsn=2

5. Zhang YJ, Wang ZH, Li CH, et al. The application of microvascular anastomotic coupler in vascular anastomosis of free tissue flap for reconstruction of defect after head

\section{doi: $10.21037 /$ fomm-21-2}

Cite this article as: Ho $M$, Schache A, Nugent $M$, Hazari A. Lessons learned from the multidisciplinary UK National Flap Registry initiative and plans for the future. Front Oral Maxillofac Med 2023;5:16. and neck cancer resection. Zhonghua Er Bi Yan Hou Tou Jing Wai Ke Za Zhi 2017;52:702-6.

6. Ho MW, Nugent M, Puglia F, et al. Results of flap reconstruction: categorisation to reflect outcomes and process in the management of head and neck defects. Br J Oral Maxillofac Surg 2019;57:935-7. 